\title{
ICS reinforcement of DRL behavior in the rat
}

\author{
JOHN A. OWENS ${ }^{1}$ AND DAVID L. BROWN \\ COLUMBIA UNIVERSITY
}

An albino rat was trained on a DRL schedule with intracranial stimulation (ICS) as a reinforcer. Reinforcement was contingent upon completion of a chain of responses similar to the chain of responses required for water reinforcement (pressing a bar and licking a dipper). The results support the generalization that ICS acts essentially like food or water reinforcement when the experimental paradigms are comparable.

Many researchers find that electrical intracranial stimulation (ICS) has different properties from the conventional reinforcers, food and water. Behavior extinguishes rapidly, response rate is high, and high reinforcement density schedules (reinforcements close together in time) must be employed (Morgan 1965). Howarth \& Deutsch (1962) hypothesize that ICS serves the dual role of rewarding the behavior that preceded it and motivating $S$ to earn more ICS. The rapid extinction of ICS-supported behavior is said to be due to a rapid decay of positive motivation. Ball (1965) agrees that each ICS furnishes motivation (which decays over time) for future behavior, but suggests that 'ICS reinforces the animal by reducing negative stimuli produced by the aftereffects of the preceding ICS." Both these theories would predict that low density ICS schedules would not support behavior.

In many classes of experiments, ICS would be more convenient than food or water, if it can be shown to affect behavior in similar ways. Intracranial reinforcement does not lead to satiation, so session lengths can be extended, and the onset, intensity, and duration of reinforcement can be precisely controlled.

Brady \& Conrad (1960) rewarded monkeys with sugar pellets on odd days and ICS on even days for criterion responses on a DRL $20 \mathrm{sec}$ schedule. On this schedule (differential reinforcement of low rates of responding) a criterion response is one which is preceded by a 20 sec period of no responding. They found that although DRL timing behavior on ICS days deteriorated considerably when the stimulation was delivered in the globus pallidus, no deterioration was apparent when stimulation was presented in the medial forebrain bundle (MFB). The existence of interpolated training for food weakens the apparent conclusion that ICS delivered to the MFB serves the same function as appetitive rewards.

Gibson, Reid, Sakai, \& Porter (1965) point out that there is a crucial difference between ICS and conventional reward paradigms. In most ICS experiments, the criterion response immediately produces the reinforcement. With conventional reinforcement, the criterion response is but the first member of a chain of responses which leads to reinforcement. The $S$ must leave the bar, approach the food or water, and consume the reward. When a chain of responses (pressing the bar then contacting a dipper) was required, no essential differences between ICS and sugar water rewards were noted in either acquisition or extinction. When only a single-element chain was required (licking the dipper), they found rapid extinction both for ICS and for sugar water reinforcement.

Pliskoff, Wright \& Hawkins (1965) report that using ICS, a DRL 3 min schedule can be maintained with a two element chain. For each completion of the 3 min DRL requirement (on a fixed bar), Ss were allowed 20 ICS reinforcements on a retractable bar. It is not clear in their paper why 20 reinforcements were considered necessary.

The purpose of the present study was to determine (1) if DRL responding with a high criterion can be supported by a single ICS at the end of a two element chain in a nondeprived rat, and (2) if the timing behavior resembles that obtained with conventional rewards.

\section{Method}

The $S$ was a 150 day old male albino rat, with a chronically implanted bipolar electrode (Plastic Products) aimed at the lateral hypothalamus. The animal was pretrained to bar press for ICS in a testing chamber distinctly different from the experimental chamber. During this pretraining period, the current level which maintained the highest rate of bar pressing was determined. This level (170 microamperes peak-to-peak, $60 \mathrm{~Hz}, .5 \mathrm{sec}$ duration) was used for all subsequent reinforcements. The $\mathrm{S}$ had ad lib access to food and water at all times except when in the testing chamber.

The testing chamber was $12 \times 7-1 / 2 \times 15$ in. high. A water dipper was mounted in the center of one wall $1-1 / 2$ in. above the stainless steel grid floor. When the dipper was activated it was displaced downward making a loud "clunk" but returned dry since the water tray was empty. A 1-in. diameter brass circular touch button was located $1-1 / 2$ in. to the right of the dipper and $2 \mathrm{in}$. above the floor. Touch circuits (which required less than .14 microamperes to operate) were connected to the dry dipper and touch button so that dipper contacts and button contacts could be separately registered.

External circuitry was arranged so that when $S$ made a button contact which met the DRL contingency the dry dipper was activated allowing $S$ to earn one ICS by touching the dipper. Noncriterion button contacts simply reset the DRL timer. The time intervals between all button contacts were recorded. 


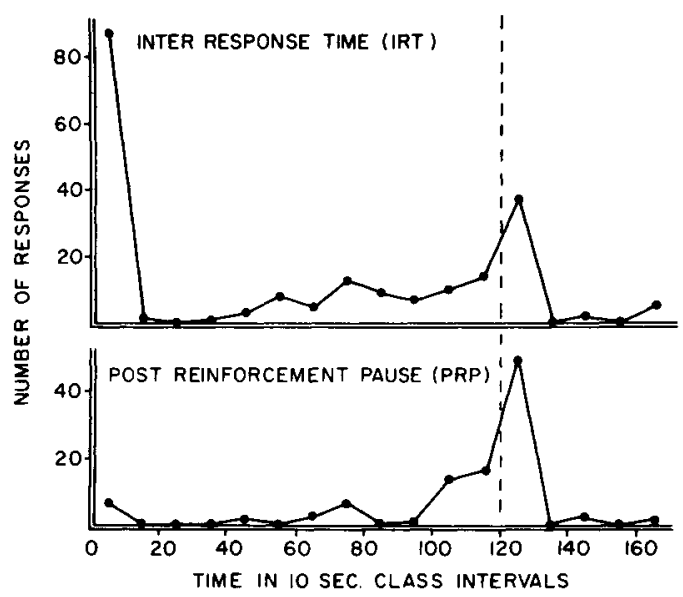

Fig. 1. Frequency distribution, in $10 \mathrm{sec}$ class intervals, of time from reinforced response to next response (PRP) and of time from unreinforced response to next response (IRT). The last point on the abscissa cumulates all times longer than $160 \mathrm{sec}$. All points to the right of the vertical dashed line, represent delays which met the DRL contingency.

The $S$ was allowed to earn between 130 and 170 reinforcements each day. If more than $20 \%$ of the responses met the DRL contingency, the contingency was raised on the following day. In this manner the contingency was raised from DRL $3 \mathrm{sec}$ to DRL $120 \mathrm{sec}$ over a period of 27 days.

\section{Resulis and Discussion}

Data from the 10th day of DRL $120 \mathrm{sec}$ is shown in Fig. 1. Postreinforcement pauses (PRPs) are defined as the time from a reinforced button contact to the next button contact. Inter-response times (IRTs) do not include the PRPs, and are defined as the time from an unreinforced button contact to the next button contact. Data from the first 100 responses (32 PRPs, 68 IRTs) are not included to avoid warm up effects. Both distributions show peaks in the 120-130 sec interval. This accuracy of response timing shows that single ICS reinforcements are just as capable of supporting DRL responding as are conventional reinforcements.

There is a characteristic peak in the shortest interval in the IRT distribution but not in the PRP distribution. Thus ICS produces the same relatively large number of short IRTs which Sidman (1956) found with appetitive rewards. The shape of the PRP distribution suggests that responses following reinforced responses are more likely to meet the DRL contingency than responses after nonreinforced responses, as has been found for DRL responding with milk reinforcement (Ferraro, Schoenfeld, \& Snapper 1965). This implies that ICS reinforcement, like conventional reinforcement, functions as a discriminative stimulus (Reynolds, 1964).

The present study has shown that ICS reinforcement of a two-element chain for a nondeprived rat will support responding on a low density schedule (reinforcements at least $2 \mathrm{~min}$ apart). If motivation for a response is generated by the previous ICS, as suggested by decay theories, this experiment implies that motivational effects still exist in some strength 2 min after an ICS reinforcement. This demonstration of such well-discriminated temporal behavior should encourage further use of ICS reinforcement with twoelement chains in the study of timing behavior where it is so important to have precise control of onset, intensity, and duration of reinforcement.

\section{References}

BALL, G. G., \& ADAMS, D. W. Intracranial stimulation as an avoidance or escape response. Psychon. Sci., 1965, 3, 39-40.

BRADY, J. V., \& CONRAD, D. C. Some effects of brain stimulation on timing behavior. J. exp. Anal. Behav., 1960, 3, 93-106.

FERRARO, D. P., SCHOENFELD, W. N., \& SNAPPER, A. G. Sequential response effects in the white rat during conditioning and extinction on a DRL schedule. J. exp. Anal. Behav., 1965, 8, 255-260.

GIBSON, W. E., REID, L. D., SAKAI, M., \& PORTER, P. B. Intracranial reinforcement compared with sugar-water reinforcers. Science, 1965, $148,1357-1359$.

HOWARTH, C. I., \& DEUTSCH, J. A. Drive decay: the cause of fast "extinction" of habits learned for brain stimulation. Science, 1962, 137, 35-36.

MORGAN, C. T. Physiological Psychology. New York: MoGraw-Hill, 1965 , p. 329.

PLISKOFF, S. S., \& HAWKINS, D. T. Brain stimulation as a reinforcer: intermittent schedules. J. exp. Anal. Behav., 1965, 8, 75-88.

REYNOLDS, G. S. Accurate and rapid reconditioning of spaced responding. J. exp. Anal Behav., 1964, 7, 273-275.

SIDMAN, M. Time discrimination and behavioral interaction in a free operant situation. J. comp. physiol. Psychol., 1956, 49, 469-473. Note

1. Now at the Department of Experimental Neurology, Isaac Albert Research Institute, Jewish Chronic Disease Hospital, Brooklyn, New York 11203. 